\title{
Role of physical exercise type in olfactory deterioration in ageing*
}

\author{
Chenping Zhang, Danyang Li, Xiaochun Wang \\ Shanghai University of Sport, 399 Chang Hai Road, Shanghai, 200438, P.R. China
}

Rhinology 58: 2, 145 - 151, 2020

https://doi.org/10.4193/Rhin19.274

*Received for publication:

August 12, 2019

Accepted: December 15, 2019

\begin{abstract}
Background: Although ageing and neurodegenerative diseases, including Alzheimer disease, have been associated with olfaction impairment, studies exploring how to ameliorate this impairment are limited. The aim of the present study was to determine the effect of various types of physical exercise on olfaction decline in ageing.
\end{abstract}

Methodology: 99 healthy community-dwelling participants (85 women; mean [SD] age, 62.5 [5.7] years) were included. All the participants were required to complete the tests consisting of a questionnaire, cognitive test and olfaction test.

Results: Odor identification scores for participants who exercised regularly for more than 1 year ( $>3$ times/wk; $>30$ min each time) were significantly higher than those for non-exercisers, and odor detection threshold scores were significantly higher in the exercisers. Both odor threshold and odor identification scores for those who exercised by practicing taiji (tai chi), dancing, or running were significantly better than those for participants who exercised by walking or who did not exercise.

Conclusion: Compared with those among older people who did not exercise, measures of olfaction among older adults who exercised were better, and the type of physical exercise mattered. Therefore, if physical exercise intervention is suggested to prevent or delay olfactory deterioration in older adults, the type of physical exercise should be considered.

Key words: ageing, Alzheimer disease, physical exercise, odor identification, odor threshold

\section{Introduction}

As suggested by Martzke et al., odor identification and the olfactory threshold sensitivity belong to different olfactory processing systems, the former is a function of the central nervous system, whereas the latter is a function of the peripheral nervous system ${ }^{(1)}$. Both odor identification and odor sensitivity impairment are highly associated with neurodegenerative diseases, such as Alzheimer disease (AD). Numerous studies have demonstrated that odor identification is significantly impaired in persons with $\mathrm{AD}$ compared with that in their healthy counterparts ${ }^{(2)}$. The olfactory threshold sensitivity is also profoundly affected in $A D^{(3)}$, with the degree of threshold deterioration correlated with the degree of dementia ${ }^{(4)}$.

Although much research has been conducted examining olfactory function as a biomarker of $A D$, studies investigating how to prevent further decline of olfaction are limited. The few studies demonstrating that regular physical exercise improves olfactory function have focused on odor identification ${ }^{(5,6)}$, but the effect of regular physical exercise on the odor detection threshold was not robust. Schubert et al. ${ }^{(7)}$ found that regular physical exercise was correlated with a reduced risk of having a higher threshold, but suggested that physical exercise may not have a linear relationship with the olfaction threshold. In addition, to the best of our knowledge, none of the previous studies took into account the type of physical exercise. Therefore, the aims of the present study were twofold: 1) to determine whether physical exercise affects measures of olfactory function and 2) to examine whether different types of physical exercise play a role in the amelioration of olfactory function decline in older persons. 


\section{Material and methods}

\section{Participants}

Because the ability of humans to identify odors begins to decline in the fifth decade of life ${ }^{(8)}$, we recruited participants who were more than 50 years old for the present study. Individuals reporting a history of any of the following conditions were excluded from the study: neurologic disease (e.g., brain tumor, Parkinson disease), local respiratory tract disorder (e.g., nasal surgery, active rhinitis or sinusitis), and psychiatric disorder (e.g., depression). All participants were asked about their history of alcohol use and cigarette smoking.

Candidates who exercised more than 3 times per week $(>30$ $\mathrm{min}$ (episode) were assigned to the exercise group, and those who did not engage in any exercise were assigned to the noexercise group.

All procedures were performed according to the Declaration of Helsinki and approved by the Shanghai University of Sport Ethics Committee (Shanghai, China). Written informed consent was obtained from all participants prior to any examination.

\section{Procedure}

The procedure consisted of a questionnaire, cognitive tests, and olfactory tests and took 1 to 2 hours for each participant to complete. Participants could break at any time after a test. The order in which the 3 tests - the Mini-Mental State Examination (MMSE) cognitive test, the odor identification test, and the odor threshold test - were conducted was counterbalanced, but the order in which the participant personal information questionnaire was given was not counterbalanced.

\section{Participant personal information questionnaire}

Participant sex, age, height, weight, smoking and drinking history, educational level, history of surgical procedures and drug use, regular physical exercise habits, and years engaged in regular physical exercise were collected using a questionnaire. For details about the questionnaire, see the Supplementary Material online.

\section{Cognitive test}

The Chinese version of the MMSE ${ }^{(9)}$ was administered to examine the cognitive function of the participants. The MMSE is a 30-point questionnaire that takes 5 to 10 minutes to complete. It examines the following functions: registration (via repeating named prompts), attention and calculation, recall, language, ability to follow simple commands, and orientation.

\section{Odor identification test}

To reduce the influence of cultural differences on the results, we used the Special Identification Test of the "Sniffin' Sticks" test (Burghart, Wedel, Germany), which is the Chinese version, to exam olfactory identification among the participants. The test was conducted as described by Hummel et al. ${ }^{(10)}$. Both nostrils were assessed for each participant in a well-ventilated room. The test included 16 odorants. Because people in China may not be familiar with 3 of the odorants in the original test, they were replaced by more familiar odorants: (No. 3) chocolate replaced cinnamon; (No. 8) ginger replaced turpentine; and (No. 12) soya sauce replaced cloves. Possible scores ranged from 0 to 16, with lower scores indicating a lower ability to identify odors.

\section{Odor threshold test}

Odor threshold was examined among the participants using the Sniffin' Sticks test with n-butanol as the odorant. The test was conducted for both nostrils in a well-ventilated room as described by Hummel et al. ${ }^{(10)}$. We prepared 16 dilutions of $n$-butanol in a geometric series. Three pens - 1 with odorant and 2 without any odorant (blank pens) - were presented to the participants for each dilution of $n$-butanol. Participants were scored using a 3-alternative forced-choice staircase paradigm. Possible scores ranged from 0 to 16 , with lower scores indicating a high smell detection threshold.

\section{Statistical analysis}

Data were analyzed using SPSS, version 22.0 (SPSS Inc., Chicago, IL, USA). For continuous variables (e.g., age, MMSE score, odor identification score, and odor threshold score), independentsample $t$ tests were conducted to evaluate the difference between scores in the exercise group and those in the no-exercise group. The chi-squared test was used to analyze dichotomous (categorical) variables (e.g., male or female sex; smoking or no smoking) when examining the differences in scores between participants in the exercise group and those in the no-exercise group. An analysis of variance (ANOVA) followed by Dunnett post hoc tests were applied to compare the main effect of different physical exercise types on odor identification and odor threshold. Pearson correlation coefficient was used to assess correlational analyses. Data are displayed as mean \pm SD. A 2-sided P $<0.05$ was considered statistically significant.

\section{Results}

\section{Study population}

In total, 108 healthy community-dwelling participants were enrolled in this study. Among the 108 enrolled participants, 99 were included in the final analysis, of which 85 (85.8\%) were women and the mean (SD) age was 62.5 (5.7) years. We exclude 9 participants from the analysis because 3 participants did not complete all of the tests, 3 participants admitted that they had an upper respiratory tract infection after completing all the tests, and 3 participants reported that they had a cold during the testing. Thus, 57 participants were included in exercise group, and 42 participants were included in the no-exercise group. There were no significant differences in age, sex, educational level, 
Table 1. Demographic characteristics and mmse, odor identification, and odor threshold scores among participants in the exercise and noexercise groups

\begin{tabular}{|lccc|}
\hline \multicolumn{1}{|c}{ Variable } & $\begin{array}{c}\text { Exercise } \\
(\mathbf{n}=\mathbf{5 7})\end{array}$ & $\begin{array}{c}\text { No Exercise } \\
(\mathbf{n}=\mathbf{4 2})\end{array}$ & P Value \\
\hline Age (mean \pm SD), y & $62.5 \pm 5.2$ & $62.4 \pm 6.4$ & 0.93 \\
\hline $\begin{array}{l}\text { Female, No. }(\%) \\
\text { Smoker, No. }(\%)\end{array}$ & $1(0.02 \%)$ & $1(0.02 \%)$ & $>.99$ \\
\hline $\begin{array}{l}\text { Educational level, } \\
\text { mean } \pm \text { SD, y }\end{array}$ & $7.9 \pm 4.1$ & $7.4 \pm 3.6$ & 00.50 \\
$\begin{array}{l}\text { MMSE score, } \\
\text { mean } \pm \text { SD }\end{array}$ & $25.6 \pm 3.8$ & $25.5 \pm 3.0$ & 0.37 \\
$\begin{array}{l}\text { Odor identification } \\
\text { score, mean } \pm \text { SD }\end{array}$ & $12.0 \pm 2.0$ & $9.9 \pm 2.9$ & $<0.001$ \\
$\begin{array}{l}\text { Odor threshold } \\
\text { score, mean } \pm \text { SD }\end{array}$ & $7.3 \pm 2.3$ & $4.9 \pm 2.3$ & $<0.001$ \\
\hline
\end{tabular}

Note. MMSE represents Mini-Mental State Examination.
Figure 1. Odor detection threshold scores and odor identification scores among participants who exercise regularly and those who do not exercise.

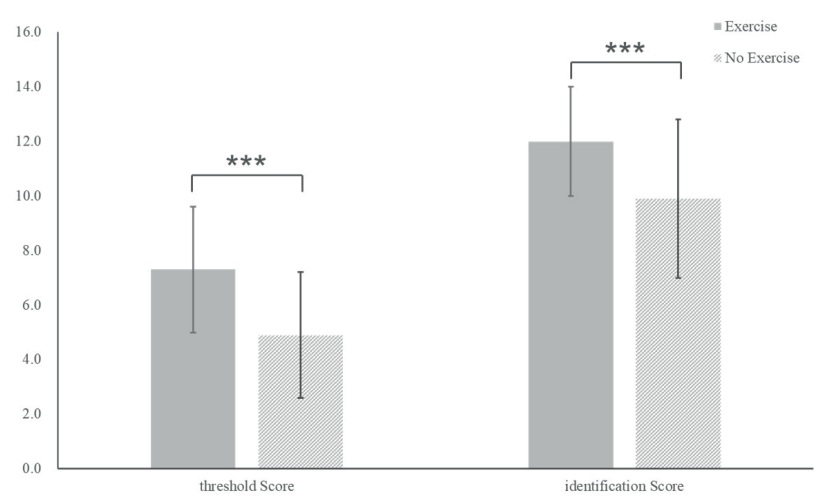

MMSE score, or smoking status either between the exercise and no-exercise groups or among the different exercise types (Table 1). None of the participants reported heavy alcohol use, and all the participants in the exercise group reported exercising for more than 1 year.

In the exercise group, 20 participants (35.1\%) practiced taiji (also known as tai chi), 14 participants (24.6\%) were dancers, 11 participants (19.3\%) were walkers, and 12 participants (21.0\%) were runners.

\section{Psychophysical test results for the exercise and no-exercise groups}

There was no significant difference in MMSE scores between the exercise group and the no-exercise group, although the MMSE scores were positively correlated with educational level $(r=$ $0.75, \mathrm{P}<0.001)$. The mean (SD) odor identification score for the exercise group (12.0 [2.0]) was significantly higher than that for the no-exercise group (9.9 [2.9]) $(\mathrm{P}<.001)$. The odor threshold score was also significantly different between these 2 groups, with the mean (SD) odor threshold scores were higher in the exercise group (7.3 [2.3]) than in the no-exercise group (4.9 [2.3]) $(P<0.001)$ (Figure 1).

\section{Olfactory test results for the types of physical exercise} Using ANOVA followed by Dunnett post hoc tests, a significant main effect of physical exercise type was found for odor identification and also for odor detection threshold (Figure 2).

The mean (SD) odor identification scores were 12.1 (1.3) for taiji practioners, 12.3 (1.1) for dancers, 12.8 (1.5) for runners, 10.2 (3.2) for walkers, and 9.9 (2.9) for those who did not exercise. The mean odor detection threshold scores were 8.5 (1.6) for taiji practioners, 8.1 (1.7) for dancers, 7.4 (2.0) for runners, 4.0 (1.2) for walkers and 4.9 (2.3) for people who did not exercise The odor detection threshold and the odor identification scores for those who exercised by practicing taiji, dancing, or running were significantly higher than for those who exercised by walking or who did not exercise, indicating that compared with older people who walked for exercise or who did not exercise, those who practiced taiji, danced or ran for exercise had a lower threshold for detecting odors and were better able to identify odors (Table 2).

\section{Discussion}

Consistent with previous research ${ }^{(5,6)}$, the present study found that older people (approximately 62 years old) who exercised were better at odor identification than those who did not exercise. In addition, we found a positive influence of physical exercise on odor threshold. Altom et al. ${ }^{(11)}$ had similar finding in dogs, revealing that suitable physical activity improves odor sensitivity in dogs. However, when we explored the impact of physical exercise type, we found that not all the types of physical exercise examined in this research had a positive effect on olfactory functions. Among the examined types of physical exercise, practicing taiji, dancing, and running significantly increased olfactory function in these older participants, whereas walking did not have the same effect. Previous studies have shown that physical exercise intensity or frequency might play an important role in the magnitude of the benefits for those with $A D$ among an ageing cohort ${ }^{(12-16)}$. A longitudinal study reported that men with the lowest physical exercise intensity had 1.8 to 2.5 times worse 10-year cognitive decline than those with higher physical exercise intensity ${ }^{(12)}$. Interestingly, intensity and variation of physical exercise, rather than duration of time spent on physical exercise, have been positively correlated with cognitive function 

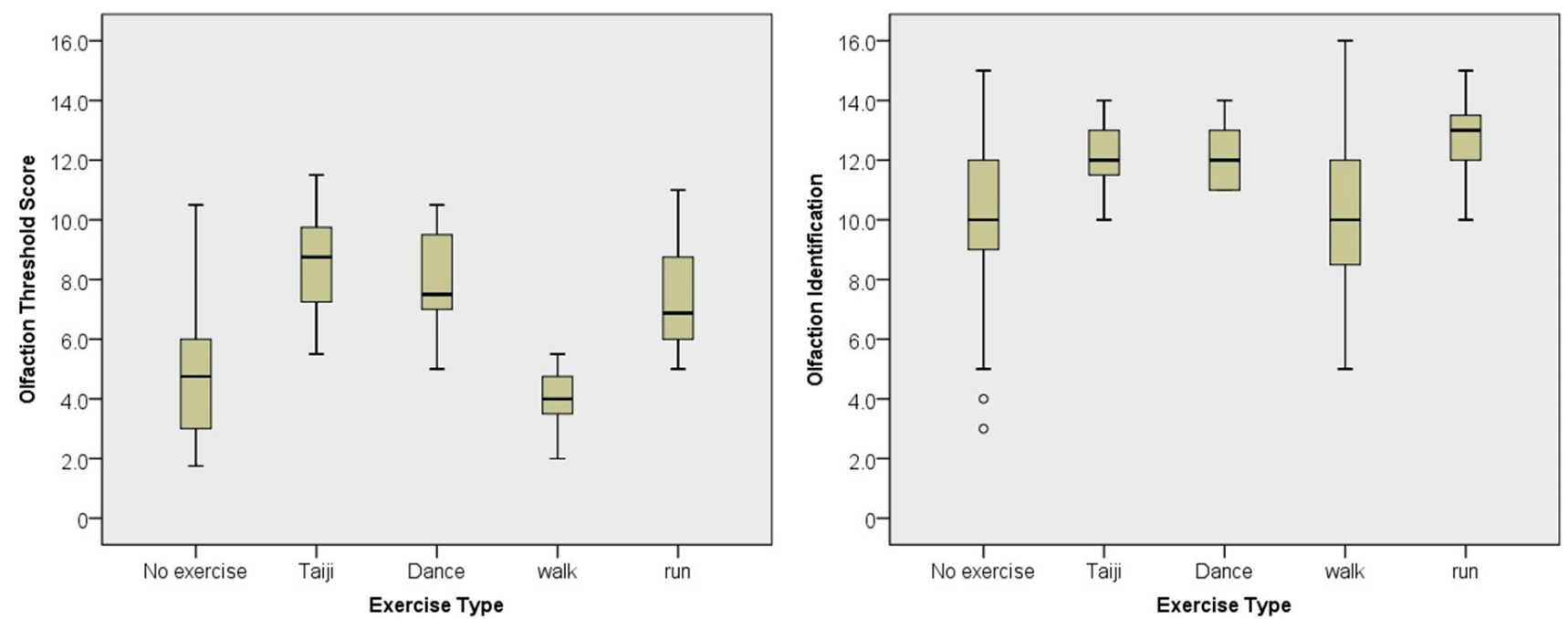

Figure 2. Odor detection threshold and odor identification scores stratified by exercise type. The boxes extend from the lower to the upper quartile values of the data, with a horizontal band at the median. The whiskers extend from the box to show the range of the data. Outlier points are those past the end of the whiskers.

Table 2. Comparison of odor threshold and odor identification scores between no exercise and 4 types of exercise.

\begin{tabular}{|c|c|c|c|}
\hline Olfactory Function & Exercise & No Exercise & P Value \\
\hline \multirow{4}{*}{$\begin{array}{l}\text { Odor threshold } \\
\text { (mean } \pm \text { SD), } \\
\text { No. of participants }\end{array}$} & Taiji $(8.5 \pm 1.6), 20$ & \multirow{4}{*}{$4.9 \pm 2.3,42$} & $<0.001$ \\
\hline & Dance $(8.1 \pm 1.7), 14$ & & $<0.001$ \\
\hline & Run $(7.4 \pm 2.0), 12$ & & 0.001 \\
\hline & Walk (4.0 \pm 1.2$), 11$ & & 0.55 \\
\hline \multirow{4}{*}{$\begin{array}{l}\text { Odor identification } \\
\text { (mean } \pm \text { SD), } \\
\text { No. of participants }\end{array}$} & Taiji (12.1 \pm 1.3$), 20$ & \multirow{4}{*}{$9.9 \pm 2.9,42$} & 0.003 \\
\hline & Dance $(12.3 \pm 1.1), 14$ & & 0.005 \\
\hline & Run (12.8 \pm 1.5$), 12$ & & 0.001 \\
\hline & Walk $(10.2 \pm 3.2), 11$ & & 0.99 \\
\hline
\end{tabular}

${ }^{(13)}$. The existence of a physical exercise intensity response on AD pathology has also been found in animal studies. Compared with high-intensity physical exercise for mice on a running wheel, mice who engaged in low-intensity physical exercise did not differ from controls in amyloid deposition ${ }^{(14)}$. Both a significant reduction in soluble $A \beta 42$ levels and a significant increase in numerous $A \beta$ clearance proteins occurred in an physical exercise training intensity-dependent manner in both the cortex and hippocampus of mice ${ }^{(15)}$. A study in mice found that exercising 3 times per week for 12 weeks significantly ameliorated their inflammatory markers, whereas 1 time per week for 12 weeks did not have an effect, suggesting that physical exercise frequency plays a role ${ }^{(16)}$. The present study included only participants who exercised more than 3 times per week and more than 30 minutes per episode; thus, exercise frequency was a factor for inclusion in the present study, but exercise intensity was not. Therefore, exercise intensity might have been a factor that caused walking to fail to positively influence olfactory function in elders although this hypothesis could not be explored in the present study. Abundant studies suggested that people in di- verse occupations were benefited from regular physical exercise, they had lower all-cause mortality rate and better overall health ${ }^{(17-20)}$. In those people who has better overall health, better olfactory function has been repeated found ${ }^{(21-23)}$. Based on these, we assumed that physical exercise improved participants' overall health and then may promoted their olfactory function. Besides physical exercise, regular olfactory training has been found also could significantly increase olfactory ability regardless of age and health status ${ }^{(24-27)}$. However, the present study only revealed the positive correlation between physical exercise and olfactory performance. In future research, the combination of physical exercise intervention and olfactory training should be employed to see the further relationship between them.

Although several studies have demonstrated that poor olfactory function predicts cognitive impairment ${ }^{(28)}$, no significant difference was found in cognitive function as assessed using the MMSE between the participants in the exercise group and those in the no-exercise group in present study. This apparent discrepant finding may have occurred at least in part because we used a previous clinical diagnosis of a cognitive disorder as 
an exclusion factor and we assessed cognitive function only with the MMSE, which may lack sensitivity. In addition, olfactory function is largely independent of cognitive deficits in Parkinson disease ${ }^{(29)}$, and olfaction dysfunction occurs at a very early stage of Alzheimer disease, even prior to the onset of the cognitive impairments typical with this disease ${ }^{(30)}$. Moreover, olfaction threshold was able to distinguish the elderly patients of idiopathic Parkinson's diseases and controls (31).

The exact mechanism responsible for physical exercise ameliorating, delaying, or preventing the deterioration of olfactory function in elders remains unclear. However, we speculate that one explanation might be physical-exercise-induced brain structural and processing changes that overlap with the olfactory pathway. As an integral part of the olfactory system, the hippocampus receives strong afferent input from the entorhinal cortex ${ }^{\left({ }^{32}\right)}$. A close relationship between hippocampal volume and odor identification has been detected by researchers ${ }^{(33)}$. Physical-exercise-induced increases in hippocampal volume have been well documented ${ }^{(34)}$. For example, that the size of the hippocampus increases by $12 \%$ to $16 \%$ with physical exercise was reported by Pajonk et al. ${ }^{(34)}$. In addition, physical exercise boosts hippocampal blood volume ${ }^{(35)}$ and increases brainderived neurotrophic factor levels ${ }^{(24,36)}$ and the volume of its release from the brain ${ }^{(37)}$. Moreover, the connectivity between frontal and hippocampal regions is significantly enhanced by physical exercise ${ }^{(38-40)}$.

There are some limitations that should be considered when interpreting the results of the present study. First, we used self-reported data rather than controlling the physical exercise intensity, frequency, or duration; however, we screened candidates to include those who exercised more than 30 minutes per episode, at least 3 times per week for more than 1 year. The use of self-reported data also limited our ability to categorize exercise intensity. Second, because the study population con- sisted of mainly women, there might be sex differences in the associations observed between exercise and olfactory function that could not be explored in the present study. However, latest research showed there was no significant difference between male and female in ageing ${ }^{(41)}$. Third, we didn't take the number of daily drugs taken into consideration, since it was found that numbers of drugs taken were highly associated with olfactory threshod ${ }^{(42)}$. Finally, we did not explore potential mechanisms to explain how physical exercise impacted odor detection threshold or odor identification.

\section{Conclusion}

Considering the high correlation between Alzheimer disease and olfactory function ${ }^{(43,44)}$, future studies should investigate whether physical exercise will improve olfactory function and the mechanism underpinning such a finding in participants with Alzheimer disease. Given that the present study found that the type of physical exercise may be important, when physical exercise intervention is applied to prevent, ameliorate, or delay olfactory deterioration in older adults, the type of physical exercise prescribed should be considered.

\section{Acknowledgements}

This work was supported by the National Natural Science Foundation of China (grant number 31971022).

\section{Authorship contribution}

$X W$ and $C Z$ designed the research protocol, $C Z$ and DL conducted the research, $C Z$ wrote the paper and XW had primary responsibility for the final content. All authors have read and approved the final manuscript.

\section{Conflict of interest}

All authors declared no conflict of interest.

\section{References}

1. Martzke JS, Kopala LC, Good KP. Olfactory dysfunction in neuropsychiatric disorders: Review and methodological considerations. Biol Psychiatry 1997; 42(8):721-732.

2. Quarmley M, Moberg PJ, MechanicHamilton D, et al. Odor Identification Screening Improves Diagnostic Classification in Incipient Alzheimer's Disease. J Alzheimer Dis2017; 55(4):14971507.

3. Djordjevic J, Jones-Gotman M, De Sousa $\mathrm{K}$, Chertkow $\mathrm{H}$. Olfaction in patients with mild cognitive impairment and Alzheimer's disease. Neurobiol. Aging2008;29, 693-706.

4. Murphy C, Gilmore MM, Seery CS, Salmon DP, Lasker BR. Olfactory thresholds are associated with degree of dementia in Alzheimer's disease. Neurobiol. Aging1990; 11, 465-469.
5. Rosenfeldt AB, Dey T, Alberts JL. Aerobic Exercise Preserves Olfaction Function in Individuals with Parkinson's Disease. Parkinson Dis 2016; 9725089.

6. Manestar D, Tićac R, Manestar K, et al. Postlaryngectomy olfactory rehabilitation and swimming. Collegium Antropologicum 2013; 37(4):1147-1152.

7. Schubert CR, Fischer ME, Pinto AA, Klein BEK, Klein R, Cruickshanks KJ. Odor detection thresholds in a population of older adults. Laryngoscope 2017; 127 (6): 1257 1262.

8. Zhang C, Wang X. Initiation of the agerelated decline of odor identification in humans: a meta-analysis. Ageing Res Rev 2017; 40,45-50.

9. Folstein MF, Folstein SE, McHugh PR. "Minimental status". A practical method for grading the cognitive state of patients for the clinician. J Psych Res 1975; 12 (3): 189-198.

10. Hummel T, Sekinger B, Wolf SR, Pauli E, Kobal G. "Sniffin' Sticks": olfactory performance assessed by the combined testing of odor identification, odor discrimination and olfactory threshold. Chem Senses 1997; 22(1): 39-52

11. Altom EK, Davenport GM, Myers LJ, Cummins KA. Effect of dietary fat source and exercise on odorant-detecting ability of canine athletes. Res Vet Sci 2003; 75(2):149155.

12. van Gelder BM, Tijhuis MA, Kalmijn S, Giampaoli S, Nissinen A, Kromhout D. Physical activity in relation to cognitive decline in elderly men: the FINE study. Neurology 2004;63(12):2316-2321.

13. Angevaren $M$, Vanhees $L$, Wendel-Vos $W$, et al. Intensity, but not duration, of physical activities is related to cognitive function. 
Eur J Cardiovasc Prev Rehabil. 2007;14: 825830.

14. Lazarov O, Robinson J, Tang YP, et al Environmental enrichment reduces $A B$ levels and amyloid deposition in transgenic mice. Cell 2005; 120, 701-713.

15. Moore KM, Girens R., Larson SK, et al. A spectrum of exercise training reduces soluble $A \beta$ in a dose-dependent manner in a mouse model of Alzheimer's disease. Neurobiol. Dis. 2016; 85, 218-224.

16. Haskins M, Jones TE, Lu Q, Bareiss SK. Early alterations in blood and brain RANTES and MCP-1 expression and the effect of exercise frequency in the $3 \times \mathrm{Tg}-\mathrm{AD}$ mouse model of Alzheimer's disease. Neurosci. Lett. 2016; 610, 165-170

17. Morris JN, Heady JA, Raffle PAB, et al. Coronary heart disease and physical activity at work. Lancet. 1953; 265: 1053-1057

18. Morris JN, Chave SP, Adam C, et al. Vigorous exercise in leisure-time and the incidence of coronary-heart disease. Lancet. 1973; 1:333-339.

19. Paffenbarger RS, Laughlin ME, Gima AS, et al. Work activity of longshoremen as related to death from coronary heart disease and stroke. N Engl J Med. 1970;282:1 109-1114.

20. Paffenbarger RS, Wing AL, Hyde RT et al. Physical activity as an index of heart attack risk in college alumni. Am J Epidemiol. 1978;108:161-175.

21. Liu B, Luo Z,Pinto JM, et al. Relationship Between Poor Olfaction and Mortality Among Community-Dwelling Older Adults: A Cohort Study. Ann Intern Med, 2019.

22. Pinto JM, Wroblewski KE, Kern DW, Schumm LP, McClintock MK. Olfactory dysfunction predicts 5-year mortality in older adults. PLoS One, 2014. 9(10): e107541.

23. Mackay-Sim A, Johnston ANB, Owen C, Burne THJ. Olfactory ability in the healthy population: reassessing presbyosmia. Chem Senses, 2006. 31(8): 763-771.

24. Hummel T, Whitcroft KL, Andrews $P$, et al. Position paper on olfactory dysfunction. Rhinology, 2017. 54(Supplement 26): 1-30.

25. Schriever VA, Lehmann S, Prange J, Hummel T. Preventing olfactory deterioration: olfactory training may be of help in older people. J Am Geriatr Soc, 2014. 62(2):
384-386.

26. Damm M, Pikart LK, Reimann H. Olfactory training is helpful in postinfectious olfactory Ioss: a randomized, controlled, multicenter study. Laryngoscope, 2014. 124(4): 826-831.

27. Sorokowska A, Drechsler E, Karwowski M, Hummel T. Effects of olfactory training: a meta-analysis. Rhinology, 2017. 55(1): 17-26.

28. Devanand D P, Lee S, Manly J, et al. Olfactory deficits predict cognitive decline and Alzheimer dementia in an urban community. Neurology 2015; 84(2):182-189.

29. Doty RL, Riklan M, Deems DA, Reynolds C Stellar S. The olfactory and cognitive deficits of Parkinson's disease: evidence for independence. Ann Neurol. 1989; 25:166-171.

30. Devanand DP, Michaels-Marston KS, Liu X et al. Olfactory deficits in patients with mild cognitive impairment predicts Alzheimer's disease at follow-up. Am J Psychiatry 2000; 157:1399-1405.

31. Foguem C, Lemdani M, Huart C. Parkinson disease in eldery patients: lessons from odour detection thresholds on olfacto-trigeminal interaction. Rhinology. 2018;56(2):127-32.

32. Price JL. Olfaction. In: Paxinos G, Mai JK, eds The Hu- man Nervous System. San Diego: Elsevier Academic Press; 2004:1197-1211.

33. Growdon ME, Schultz AP, Dagley AS, et al. Odor identification and Alzheimer disease biomarkers in clinically normal elderly. Neurology 2015; 84(21):2153-2160.

34. Pajonk F-G, Wobrock T, Gruber O, et al. Hippocampal plasticity in response to exercise in schizophrenia. Arch. Gen. Psychiatry 2010; 67, 133-143.

35. Pereira AC, Huddleston DE, Brickman AM, et al. An in vivo correlate of exercise-induced neurogenesis in the adult dentate gyrus. Proc Nat Acad Sci (USA) 2007;104 (13):56385643.

36. Erickson KI, Miller DL, Roecklein KA. The aging hippocampus: interactions between exercise, depression, and BDNF. Neuroscientist 2012; 18:82e97.

37. Seifert $T$, Brassard $P$, Wissenberg $M$, et al Endurance training enhances BDNF release from the human brain. Am J Physiol Regul Integr Comp Physiol. 2010; 298(2): R372R377.
38. Burdette JH, Laurienti PJ, Espeland MA, et al. Using network science to evaluate exerciseassociated brain changes in older adults. Front Aging Neurosci. 2010; 2:23.

39. Voss MW, Erickson Kl, Prakash RS, et al. Functional connectivity: a source of variance in the association between cardiorespiratory fitness and cognition? Neuropsychologia 2010; 48(5):1394-1406.

40. Voss MW, Prakash RS, Erickson Kl, et al. Plasticity of brain networks in a randomized intervention trial of exercise training in older adults. Front Aging Neurosci. 2010; 2:32.

41. Wang $X$, Zhang C, Xia X, Yang Y, Zhou C. Effect of gender on odor identification at different life stages: a meta-analysis. Rhinology 2019; 57: 5, 322-330.

42. Ottaviano G, Savietto E, Scarpa B, et al. Influence of number of drugs on olfaction in the elderly. Rhinology. 2018;56(4):351-7.

43. Lafaille-Magnan ME, Poirier J, Etienne P, et al. Odor identification as a biomarker of preclinical AD in older adults at risk. Neurology 2017; 89:1-9.

44. Schiffman SS, Graham BG, Sattely-Miller EA, Zervakis J, Welsh-Bohmer K. Taste, smell and neuropsychological performance of individuals at familial risk for Alzheimer's disease. Neurobiol Aging 2002;23(3):0-404

Professor Xiaochun Wang

School of Psychology

Shanghai University of Sport

399 Chang Hai Road

Shanghai

200438, P.R. China

Tel/Fax: 00862151253152

E-mail: wangxiaochun@sus.edu.cn

This paper contains supplementary materials online: at www.rhinologyjournal.org 


\section{SUPPLEMENTARY INFO}

\section{Participant Personal Information Questionnaire}

Dear Participants,

Thank you for your time completing this questionnaire. All the information will be strictly kept confidential. Please answer all the question with honest. Thank you again.

Q1. Gender: Male ( ) Female ( )

Q2. Date of Birth:

Q3. Height (cm):

Q4. Weight (kg):

Q5. Do you smoke or used to smoke? If yes, please specify.

Yes ( ) Please specify how much cigarette taken. No ( )

Q6. Do you drink or used to drink? If yes, please specify.

Yes ( ) Please specify daily volume consumed. No ( )

Q7. Have you got serious disease in recent 5 years (request surgery)?

Yes ( ) Please specify. No ( )

Q8. Are you taking drugs?

Yes ( ) Please specify.

No ( )

Q9. How about your sleep?

A Good B Not bad, may sleepless occasionally

C Very bad, sleepless most of the time

Q10. What's your education?

$A \leq 6$ years $\quad B$ 7 -9 years $\quad$ C $10-12$ years $\quad D \geq 13$ years

Q11. How many times you exercise in a week ? $(\geq 30 \mathrm{~min} /$ time)

A $>5$ times B 3-5 times C 1-2 times D No regular exercise

Q12. How long have you been exercise?

$A<1$ year $B 1-3$ years $C>3$ years 